\title{
Pápulas milimétricas hiperpigmentadas en piernas
}

\section{Hyperpigmented millimetric papules on legs}

\author{
Alberto Ramos-Garibay, ${ }^{*}$ Juan Ramón Trejo-Acuña, ${ }^{\ddagger}$ \\ Myrna del Carmen Rodríguez-Acar, ${ }^{\S}$ Brenda Mariel Porras-Zamora $\llbracket$
}

\section{CASO CLÍNICO}

Se trata de una mujer de 37 años de edad, empleada, originaria y residente de la Ciudad de México, quien acude a valoración en la consulta del Centro Dermatológico
«Dr. Ladislao de la Pascua» por presentar lesiones que aparecieron 11 años antes, intensamente pruriginosas. Sin antecedentes de importancia para su padecimiento. Negó tratamientos previos. A la exploración física se observa dermatosis diseminada a extremidades

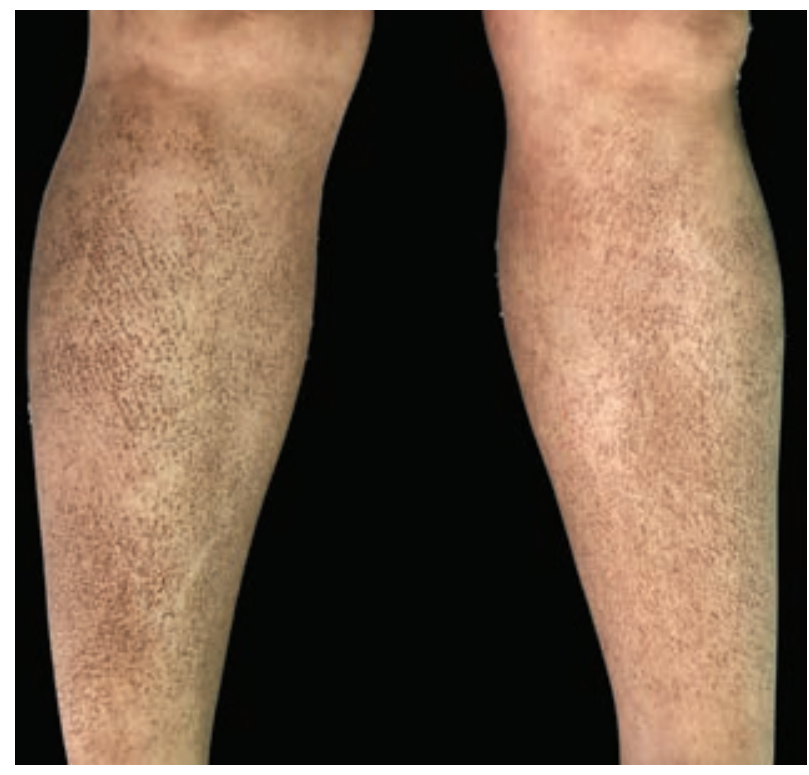

Figura 1: Pápulas milimétricas, color marrón oscuro, agrupadas en un patrón de aspecto reticular que afectan miembros inferiores.

* Dermatopatólogo.

₹ Dermatooncólogo.

$\S$ Jefe de la Consulta Externa.

" Residente del segundo año de Dermatología.

Citar como: Ramos-Garibay A, Trejo-Acuña JR, Rodríguez-Acar MC, Porras-Zamora BM. Pápulas milimétricas hiperpigmentadas en piernas. Rev Cent Dermatol Pascua. 2021; 30 (3): 166-169. https://dx.doi.org/10.35366/103832 


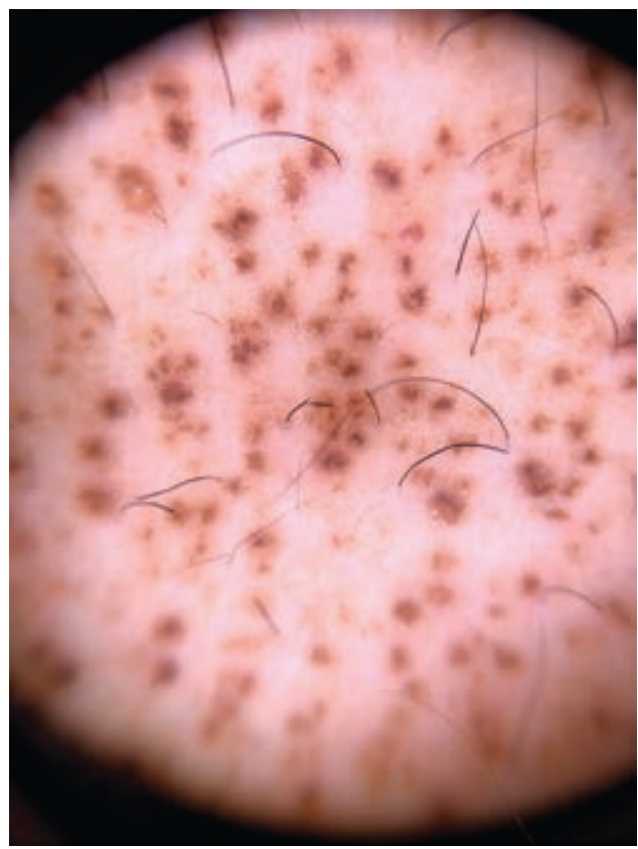

Figura 2: Dermatoscopía. Se observa depósito de pigmento con distribución aleatoria.

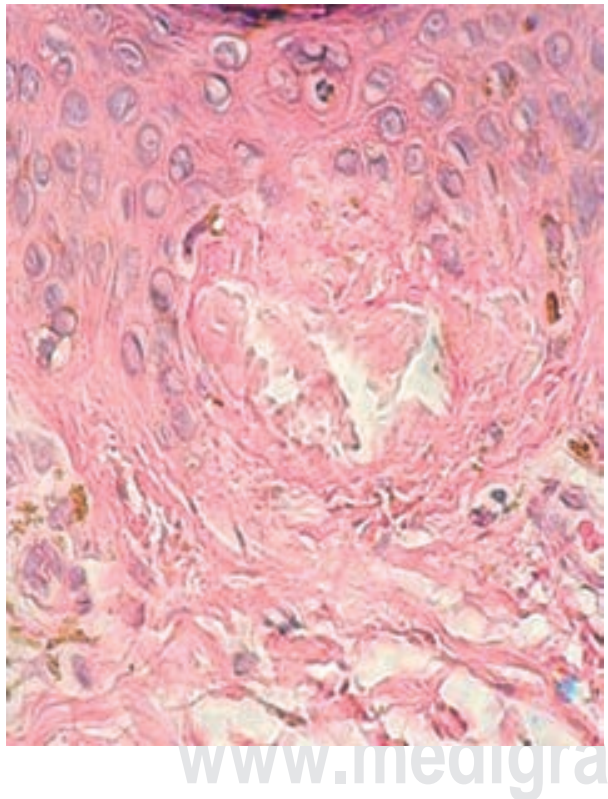

inferiores, de las que afecta cara anterior de piernas casi en su totalidad, dejando sólo algunas áreas de piel sana, de aspecto monomorfo, monolesional, constituida por incontables pápulas milimétricas, color marrón oscuro, agrupadas en un patrón de aspecto reticular. De evolución crónica y muy pruriginosa (Figura 1). A la dermatoscopía se observa depósito de pigmento

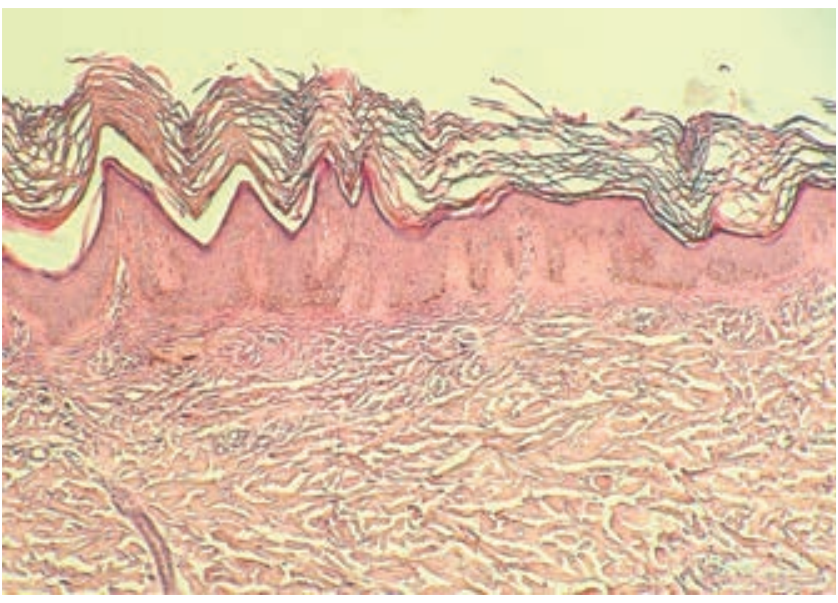

Figura 3: Tinción de rutina (H\&E 4x).

Figura 4:

\section{A mayor aumento, tinción} de rutina (H\&E 10x).

con distribución aleatoria (Figura 2). En el resto de piel y anexos no se encontraron datos patológicos. Se realiza biopsia incisional, cuyos cambios histológicos se observan en las Figuras 3 y 4.

Con los datos proporcionados anteriormente, ¿cuál sería su diagnóstico clínico presuntivo? 
Ramos-Garibay A y cols. Pápulas milimétricas hiperpigmentadas en piernas

\section{DIAGNÓSTICO: AMILOIDOSIS CUTÁNEA PRIMARIA LOCALIZADA}

Se conoce como amiloidosis a un grupo de patologías caracterizadas por el depósito extracelular de proteínas en los diversos tejidos del organismo. Es discretamente más frecuente en hombres, y la edad promedio de presentación es entre la cuarta y quinta décadas de la vida. ${ }^{1}$ Se le clasifica como sistémica o localizada, dependiendo en dónde se deposite dicho material, o primaria y secundaria, si se asocia o no con otras patologías. ${ }^{2}$

La amiloidosis cutánea primaria localizada (ACPL) se subdivide en tres tipos: macular, liquenoide y nodular, dependiendo de las características clínicas que predominen. Existe controversia para definir cuál subtipo es el más común; sin embargo, las últimas publicaciones indican que el tipo nodular se presenta en $65 \%$ de los casos, seguido por el liquenoide, luego el macular, y por último el bifásico, el cual se considera formado por la coexistencia de los dos últimos. ${ }^{3}$

El subtipo nodular se manifiesta con una o varias neoformaciones de aspecto nodular acompañadas de atrofia y telangiectasias en su periferia. ${ }^{4}$ Aunque su causa aún no se ha definido por completo, se sugiere que esta variante en especial es secundaria a la disfunción local de las células plasmáticas a nivel local.5,6

Los subtipos macular y liquenoide se diferencian por la lesión elemental predominante; no obstante, comparten las mismas características fisio e histopatológicas. ${ }^{2}$ La primera se manifiesta como manchas hiperpigmentadas color marrón oscuro-grisáceo, de distribución simétrica y aspecto reticulado, que frecuentemente se ubican en tórax superior (tanto anterior, como posterior), y en raras ocasiones en cara y extremidades superiores. ${ }^{1,7}$

En contraste, la variedad liquenoide se presenta como pápulas milimétricas hiperpigmentadas, que pueden o no acompañarse de escama, y son intensamente pruriginosas; predominan en la superficie extensora de las extremidades, tanto superiores como inferiores. ${ }^{2}$

Por el momento, no se han detectado patrones dermatoscópicos específicos descritos para esta patología; no obstante, existen comunicaciones que coinciden en haber detectado la presencia de estructuras hiperpigmentadas de bordes difusos. ${ }^{6}$

La histopatología de ambos tipos muestra depósitos de amiloide agrupados en pequeños glóbulos que ocupan la dermis, así como incontinencia del pigmento. ${ }^{2}$ En la mayoría de los casos con el empleo de las tinciones de rutina es suficiente para realizar el diagnóstico; ahora bien, existen otras tinciones, como rojo congo, las cuales son más específicas y definiti- vas. Con esta última técnica el material amiloide se tiñe de color verde birrefringente utilizando el microscopio de luz polarizada. ${ }^{1}$

La teoría fisiopatológica más aceptada sugiere que la apoptosis inapropiada de los queratinocitos genera degeneración filamentosa de éstos, lo cual culmina con el depósito de material amiloide. Hasta ahora no se ha detectado un factor específico que desencadene esta patología; con todo, la mayoría de los pacientes comparte el antecedente de traumatizar superficialmente su piel en forma repetitiva al utilizar sus propias uñas o dispositivos de material sintético para disminuir el prurito. En general, la evolución es larga, mayor a cinco años. ${ }^{8}$

Dentro de los principales diagnósticos diferenciales de la amiloidosis macular o liquenoide se encuentran el liquen simple crónico, el liquen hipertrófico o el mixedema pretibial. La variedad nodular debe distinguirse de la mucinosis papular, del prurigo nodular y del pseudolinfoma. $^{2}$

Los objetivos del tratamiento para esta patología consisten en aliviar el prurito y tratar de remover los depósitos de amiloide de la dermis. Para el primero se han utilizado diversos agentes como inhibidores de calcineurina (tacrolimus al $0.1 \%$ ) o análogos de vitamina D (calcipotriol), para el segundo se han hecho pruebas con retinoides tópicos y sistémicos, inmunomoduladores como la ciclosporina y agentes más invasivos como el láser $\mathrm{Nd}$ :Yag o $\mathrm{CO}_{2} \cdot{ }^{1}$

Actualmente, existen diversas publicaciones de pacientes que han evolucionado con remisión casi total de las lesiones con la administración de isotretinoína oral a dosis de $20-30 \mathrm{mg} /$ día sin presentar efectos secundarios, por lo que este fármaco debe considerarse como alternativa terapéutica en aquellos pacientes con mala respuesta a tratamiento tópico. ${ }^{4}$

La amiloidosis cutánea primaria localizada se ha considerado como un padecimiento benigno, debido a que únicamente $1 \%$ de los pacientes que la padecen han evolucionado a enfermedad sistémica, y sólo $9 \%$ tiene riesgo de presentar más lesiones a lo largo de la vida; sin embargo, la amplia gama de opciones terapéuticas refleja la complejidad para tratarla y la ausencia de un procedimiento de elección. ${ }^{1}$

\section{COMENTARIO}

La amiloidosis cutánea localizada es una patología poco común, cuya etiología aún no ha sido bien definida. A pesar de que en la actualidad existen numerosos auxiliares que ayudan a confirmar el diagnóstico 
clínico presuntivo, ninguno ha superado al estudio histopatológico. Aunque parece una entidad fácilmente reconocible, es fundamental realizar dicho estudio, debido a los numerosos diagnósticos diferenciales que deben descartarse.

Es importante insistir a los pacientes evitar traumatismos por rascado o por el uso de dispositivos sintéticos con la finalidad de limitar la progresión de las lesiones, ya que en la actualidad no existe un tratamiento específico para esta patología. Las numerosas opciones terapéuticas poco concluyentes reflejan el reto que representa esta enfermedad para lograr la remisión parcial o total de las lesiones. En la mayoría de los casos sólo se logra lo primero, por lo que es necesario continuar haciendo estudios para mejorar el pronóstico de los pacientes con esta entidad.

La paciente del caso comunicado en este artículo fue tratada inicialmente con cremas con urea al $20 \%$ y lubricación. Se le citó a los dos meses. En esa ocasión no se observó mejoría, por lo cual se decidió iniciar con isotretinoína vía oral, $20 \mathrm{mg}$ al día, continuando con urea, pero ahora al $10 \%$. Se le citó en dos meses más. Desafortunadamente, la paciente ya no acudió a dicha cita, por lo cual se desconoce su estado actual.

\section{REFERENCIAS}

1. KaltoftB, Schmidt G, Lauritzen AF, Gimsing P. Primary localised cutaneous amyloidosis: a systematic review. Dan Med J. 2013; 60: A4727.

2. Calonje E, Brenn T, Lazar A, McKee P. McKee's pathology of the skin. 4th ed. Elsevier; 2012. pp. 537-540.

3. Ladizinski B, Lee KC. Lichen amyloidosis. CMAJ. 2014; 186: 532.

4. Atacan D, Ergin C, Celik G, Gonul M, Adabag A. Oral isotretinoin: a new treatment alternative for generalized lichen amyloidosis. Australas J Dermatol. 2016; 57: 246-247.

5. Heaton J, Steinhoff N, Wanner B, Krutchik M, Bountiful UT. A review of primary cutaneous amyloidosis. JAOCD. 2017; 38: 46-49.

6. Chuang YY, Lee DD, Lin CS, Chang YJ, Tanaka M, Chang YT et al. Characteristic dermoscopic features of primary cutaneous amyloidosis: a study of 35 cases. Br J Dermatol. 2012; 167: 548-554.

7. Borowicz J, Shama L, Miller R. Nodular cutaneous amyloidosis. Skinmed. 2011; 9: 316-318.

8. Bolognia J, Schaffer J, Cerroni L, Callen J, Cowen E, Hruza G et al. Dermatology. Vol. 1. 3rd ed. Elsevier; 2012. pp. 702-703.

Correspondencia:

Dr. Alberto Ramos-Garibay

Dr. Vértiz No. 464, esq. Eje 3 Sur,

Col. Buenos Aires, 06780 ,

Alcaldía Cuauhtémoc, CDMX.

Tel: 55 5519-6351

E-mail: ramosgari4400@yahoo.com 